\title{
FIXED POINTS AND MULTIPLICATIVE LEFT INVARIANT MEANS
}

\author{
BY \\ THEODORE MITCHELL(1)
}

1. Introduction. An abstract semigroup, $S$, will be said to have the common fixed point property on compacta if for each compact Hausdorff space $X$, and for each homomorphic representation $\mathscr{S}$ of $S$ as a semigroup (under functional composition) of continuous maps of $X$ into itself, there is in $X$ a common fixed point of the family $\mathscr{S}$. This paper solves the problem of characterizing those semigroups that have the above property.

E. S. Ljapin [5, Theorem 5.3, p. 66] has characterized the class of semigroups such that for each arbitrary homomorphic representation of the semigroup by transformations on any set, every transformation has a fixed point (not necessarily common) in the set. Such semigroups, which in Ljapin's terms are of the class $\pi$, are those that satisfy the condition that each element has a right zero; that is for each $s$ in the semigroup $S$, there exists $s^{\prime} \in S$ such that $s s^{\prime}=s^{\prime}$.

M. M. Day [2] characterized the class of semigroups $S$ having the following attribute, which we shall call the Day fixed point property: for each compact convex subset $K$ of each locally convex space $X$, and for each homomorphic representation $\mathscr{S}$ of $S$ as a semigroup of continuous affine maps of $K$ into itself, there is in $K$ a common fixed point of the family $\mathscr{S}$. It is shown in [2] that $S$ has the Day fixed point property if and only if $S$ has a left invariant mean.

When a semigroup $S$ has the common fixed point property on compacta, it is immediate from the definitions that $S$ has the Day fixed point property. But there exist semigroups that satisfy the second condition but not the first; an example of this is given by any nontrivial Abelian group (see Theorem 2). So each semigroup with the common fixed point property on compacta has a left invariant mean, but the converse does not hold. In the principal result of this paper, Theorem 1, it is shown that a semigroup $S$ has the common fixed point property on compacta if and only if $S$ has a multiplicative left invariant mean.

Theorem 1 also contains two purely algebraic characterizations of such a semigroup in terms of its left thick subsets. A subset $S^{\prime}$ of a semigroup $S$ is called left thick in $S$ if for any finite subset $S^{\prime \prime} \subseteq S$, there exists $s^{\prime \prime} \in S$ such that $S^{\prime \prime} s^{\prime \prime} \subseteq S^{\prime}$. A semigroup $S$ with the common fixed point property on compacta is shown to be characterized by a condition reminiscent of the proof of the Bolzano-Weierstrass

(1) Presented to the Society, April 9, 1965; received by the editors August 4, 1965. 
Theorem: any decomposition of $S$ into a finite number of subsets contains at least one left thick subset of $S$.

The relationship of semigroups of the class $\pi$ to the class of those having the common fixed point property on compacta has not been fully ascertained, however. There are semigroups in the first class that are not in the second (for example, the family of all continuous maps on the closed unit interval into itself). But it is not known if the first class includes the second.

In $\S 2$, we state preliminaries, definitions, and the nomenclature used throughout the paper. The main theorem and its corollaries are obtained in $\S 3$. $\$ 4$ presents examples and obtains additional algebraic results concerning Abelian semigroups and cancellation semigroups that have the common fixed point property on compacta.

2. Preliminaries and notation. For terminology not given here or in a later section, see Day [1]. Let $S$ be a semigroup, $m(S)$ the space of all bounded realvalued functions on $S$, where $m(S)$ has the supremum norm. An element $\mu \in m(S)^{*}$ in a mean on $m(S)$ if $\|\mu\|=1$, and $\mu(e)=1$, where $e$ denotes the constant 1 function on $S$. A mean $\mu$ is left invariant if $\mu\left(l_{s} f\right)=\mu(f)$ for all $f \in m(S)$ and $s \in S$, where the left translation $l_{s}$ of $m(S)$ by $s$ is given by $\left(l_{s} f\right) s^{\prime}=f\left(s s^{\prime}\right)$. A semigroup that has a left invariant mean is called left amenable. A mean $\mu$ is multiplicative if $\mu(f) \cdot \mu(g)=\mu(f \cdot g)$ (the pointwise product) for all $f, g \in m(S)$. The space of multiplicative means on $m(S)$ shall be designated by $\beta(S$.)

If $f \in m(S)$ and $\mu \in m(S)^{*}$, then $\mu(f)$ shall be alternatively designated by $(\mu, f)$. For each $\mu \in m(S)^{*}$, there is associated a map $\mu_{l}: m(S) \rightarrow m(S)$ given by $\left(\mu_{l} f\right) s=\left(\mu, l_{s} f\right)$, for $f \in m(S), s \in S$. For any two elements $\mu, \mu^{\prime} \in m(S)^{*}$, a product $\mu \odot \mu^{\prime} \in m(S)^{*}$ is defined by $\left(\mu \odot \mu^{\prime}, f\right)=\left(\mu, \mu^{\prime}{ }_{l} f\right)$ for $f \in m(S)$. When given the product $\odot$ and the $w^{*}$-topology of $m(S)^{*}$, then $\beta(S)$ is a semigroup and also a compact Hausdorff space (but it need not be a topological semigroup), for $\beta(S)$ is homeomorphic to the Stone-Cech compactification of the discrete set $S$. The symbol $Q$ shall denote the evaluation isomorphism of $S$ into the semigroup $\beta(S)$, where $(Q s, f)=f(s)$ for $f \in m(S), s \in S$.

\section{The main characterization.}

THEOREM 1. Let $S$ be a semigroup. Then the following are equivalent:

(a) $S$ has a multiplicative left invariant mean.

(b) For each pair of subsets $S_{1}, S_{2} \subseteq S$ such that $S_{1} \cup S_{2}$ is left thick in $S$, it follows that at least one of the subsets $S_{1}, S_{2}$ is left thick in $S$.

(c) For each finite collection of subsets $S_{i} \subseteq S$ such that $S=\bigcup_{i=1}^{n} S_{i}$, it follows that at least one of the subsets $S_{i}$ is left thick in $S$.

(d) $S$ has the common fixed point property on compacta.

Proof. (a) $\rightarrow$ (b). Let $S^{\prime}$ be left thick in $S$, where $S^{\prime}=S_{1} \cup S_{2}$. Let $\left\{S_{\gamma}\right\}$ be the family of finite subsets $S_{\gamma} \subseteq S$ directed upwards by inclusion. For each finite 
subset $S_{\gamma} \subseteq S$, there exists $s_{\gamma} \in S$ such that $S_{\gamma} s_{\gamma} \subseteq S^{\prime}$. By $w^{*}$-compactness of $\beta(S)$, the net $\left\{Q s_{\gamma}\right\}$ has a subnet $\left\{Q s_{\delta}\right\}$ which is $w^{*}$-convergent to some $\mu^{\prime} \in \beta(S)$. For any $s \in S$,

$$
l_{s}^{*} \mu^{\prime}=l_{s}^{*}\left(w^{*} \lim _{\delta}\left(Q s_{\delta}\right)\right)=w^{*} \lim _{\delta}\left(l_{s}^{*}\left(Q s_{\delta}\right)\right),
$$

where the second equality follows by $w^{*}$-continuity of $l_{s}^{*}$. Let $f_{0} \in m(S)$ be the characteristic function of $S^{\prime}$. Then

$$
\begin{aligned}
\left(l_{s}^{*} \mu^{\prime}, f_{0}\right) & =\lim _{\delta}\left(l_{s}^{*}\left(Q s_{\delta}\right), f_{0}\right)=\lim _{\delta}\left(Q s_{\delta}, l_{s} f_{0}\right) \\
& =\lim _{\delta}\left(\left(l_{s} f_{0}\right) s_{\delta}\right)=\lim _{\delta} f_{0}\left(s s_{\delta}\right)=1
\end{aligned}
$$

for each $s \in S$. By (a), $S$ has a multiplicative left invariant mean $\mu$. Let $\mu^{\prime \prime} \in \beta(S)$ be given by $\mu^{\prime \prime}=\mu \odot \mu^{\prime}$. Then $\mu^{\prime \prime}$ is a left invariant mean by [1, Corollary 2 , p. 529]. Further, for any $s \in S$,

$$
\mu_{l}^{\prime} f_{0}(s)=\left(\mu^{\prime}, l_{s} f_{0}\right)=\left(l_{s}^{*} \mu^{\prime}, f_{0}\right)=1,
$$

hence $\mu_{l}^{\prime} f_{0}=e$. Thus

$$
\mu^{\prime \prime} f_{0}=\left(\mu, \mu_{l}^{\prime} f_{0}\right)=(\mu, e)=1 .
$$

Let $f_{1}$ and $f_{2}$ be the characteristic functions of $S_{1}$ and $S_{2}$ respectively. Since $\mu$ " is multiplicative, its value on a characteristic function is zero or one. Since $\mu^{\prime \prime} f_{0}=1$, it cannot be that both $\mu^{\prime \prime} f_{1}=0$ and $\mu^{\prime \prime} f_{2}=0$. So at least one of the functions $f_{i}$, where $i=1,2$, satisfies $\mu^{\prime \prime} f_{i}=1$. But if $V$ is any subset of a left amenable semigroup $S$, then $V$ is left thick in $S$ if and only if there exists a left invariant mean on $S$ whose value on the characteristic function of $V$ is one, [6, Theorem 7]. So at least one of the subsets $S_{i}$ is left thick in $S$.

(b) $\rightarrow$ (c). $S$ is left thick in itself; the rest follows by a straightforward induction argument.

(c) $\rightarrow$ (d). Let $\eta: S \rightarrow \mathscr{S}$ be a homomorphism of $S$ onto $\mathscr{S}$, a semigroup of continuous maps of $X$ into $X$. Let $y$ be a specific point of the compact Hausdorff space $X$. First we will show that there exists a $y_{0} \in X$ such that for every open neighborhood $U$ of $y_{0}$, where $U \subseteq X$, the set $\{s \in S ;(\eta s) y \in U\}$ is left thick in $S$.

Suppose no such point $y_{0} \in X$ exists. Then for each $x \in X$, there exists an open neighborhood of $x$, the set $U_{x} \subseteq X$, such that the set $S_{x}$ defined by

$$
S_{x}=\left\{s \in S ; \eta(s) y \in U_{x}\right\}
$$

is not left thick in $S$. But the family $\left\{U_{x} ; x \in X\right\}$ is an open covering of $X$, so by compactness of $X$ there exists a finite number of elements $x(i) \in X$ such that $X=\bigcup_{i=1}^{n} U_{x(i)}$. Thus $S=\bigcup_{i=1}^{n} S_{x(i)}$, which contradicts (c). Hence such a $y_{0} \in X$ exists.

Let $\Gamma$ be the family of all finite subsets of $S$ directed upwards by inclusion, and $\Delta$ the family of all open neighborhoods of $y_{0}$ directed downwards by inclusion. 
For convenience, we refer to a finite subset of $S$ as $S_{\gamma}^{\prime \prime}$ rather than as $\gamma$ itself, and similarly to an open neighborhood of $y_{0}$ as $Y_{\delta}$ rather than as $\delta$. Further, $\left\{S_{\delta}^{\prime}\right\}$ will designate the net of left thick subsets of $S$ given by $S_{\delta}^{\prime}=\left\{s \in S ; \eta(s) y \in Y_{\delta}\right\}$. Let $\Psi=\Gamma \times \Delta$, the product directed set of $\Gamma$ and $\Delta$. For each $\psi=(\gamma, \delta) \in \Psi$, by left thickness of $S_{\delta}^{\prime}$ in $S$ there exists $s_{\psi} \in S$ such that $S_{\gamma}^{\prime \prime} s_{\psi} \subseteq S_{\delta}^{\prime}$. For any $s \in S$, the net $\left\{s s_{\psi}\right\}$ is eventually in each $S_{\delta}^{\prime}$, which implies that the net $\left\{\eta\left(s s_{\psi}\right) y\right\}$ is eventually in each open neighborhood $Y_{\delta}$ of $y_{0}$. Thus $y_{0}=\lim _{\psi}\left(\eta\left(s s_{\psi}\right) y\right)$ for all $s \in S$.

Let $s_{0}$ be a specific element of $S$. Then for all $s \in S$,

$$
\begin{aligned}
\eta(s) y_{0} & =\eta(s) \lim _{\psi}\left(\eta\left(s_{0} s_{\psi}\right) y\right)=\lim _{\psi}\left(\eta(s) \eta\left(s_{0} s_{\psi}\right) y\right) \\
& =\lim _{\psi}\left(\eta\left(s_{0} s_{\psi}\right) y\right)=y_{0},
\end{aligned}
$$

where the second equality follows by continuity of $\eta(s)$, and the third equality by the fact that $\eta$ is a homomorphism. Hence $y_{0}$ is the required common fixed point of the family $\mathscr{S}$.

(d) $\rightarrow$ (a). Choose the space $\beta(S)$, where $\beta(S)$ has the $w^{*}$-topology, to be the compact Hausdorff space $X$. For each $s \in S$, define a map $T_{s}: \beta(S) \rightarrow \beta(S)$ by $T_{s} \mu=l_{s}^{*} \mu$ for $\mu \in \beta(S)$. The set $\mathscr{S}=\left\{T_{s} ; s \in S\right\}$ forms a semigroup, homomorphic to $S$, of $w^{*}$-continuous maps of $\beta(S)$ into itself. Then by (d), there exists $\mu_{0} \in \beta(S)$ such that $\mu_{0}=T_{s} \mu_{0}=l_{s}^{*} \mu_{0}$, for all $s \in S$. Hence $\mu_{0}$ is the required multiplicative left invariant mean.

An elementary example of a semigroup that has a multiplicative left invariant mean is given by any semigroup $S$ that contains a right zero, that is an $s_{0} \in S$ such that $S s_{0}=\left\{s_{0}\right\}$. Then $Q\left(s_{0}\right)$ can be verified to be the indicated mean. A partial converse to this is given by the following result:

Corollary 1. A finite semigroup $S$ has a multiplicative left invariant mean if and only if $S$ contains a right zero.

Proof. The "if" part follows from the remark above. For the converse, $S=\bigcup_{i=1}^{n}\left\{s_{i}\right\}$, so by condition (c) of Theorem 1, there exists a left thick singleton subset $\left\{s^{\prime}\right\} \subseteq S$. Thus there exists $s^{\prime \prime} \in S$ such that $S s^{\prime \prime}=\left\{s^{\prime}\right\}$. In particular, $s^{\prime \prime} s^{\prime \prime}=s^{\prime} s^{\prime \prime}=s^{\prime}$. Therefore

$$
S s^{\prime}=S\left(s^{\prime \prime} s^{\prime \prime}\right)=\left(S s^{\prime \prime}\right) s^{\prime \prime}=\left\{s^{\prime} s^{\prime \prime}\right\}=\left\{s^{\prime}\right\},
$$

hence $s^{\prime}$ is a right zero of $S$. (Simple proofs of Corollary 1 can also be constructed by use of conditions (b) or (d) of Theorem 1.)

Corollary 2. Let $\eta$ be a homomorphism of $S$ onto $S^{\prime}$, where $S$ and $S^{\prime}$ are semigroups. If $S$ has a multiplicative left invariant mean, then so has $S^{\prime}$.

Proof. Let $S$ have property (a), hence property (d) of Theorem 1 . Let $X$ be any compact Hausdorff space and $\eta^{\prime}$ any homomorphism of $S^{\prime}$ onto $\mathscr{S}^{\prime}$, where 
$\mathscr{S}^{\prime}$ is a semigroup of continuous maps on $X$ into itself. But $\eta^{\prime} \eta$ is then a homomorphism of $S$ onto $\mathscr{S}^{\prime}$, thus $X$ contains a common fixed point of the family $\mathscr{S}^{\prime}$. Hence $S^{\prime}$ satisfies property (d), thus property (a), of Theorem 1 .

COROLlaRY 3. Let $S$ be a semigroup such that for every $s, s^{\prime} \in S$, there exists an $s " \in S$ which satisfies $s s^{\prime \prime}=s^{\prime} s$. Then $S$ has a multiplicative left invariant mean.

Proof. It follows by an induction argument that for each finite subset $S^{\prime \prime} \subseteq S$, there exists $s^{\prime \prime} \in S$ such that $S^{\prime \prime} s^{\prime \prime}=\left\{s^{\prime \prime}\right\}$, (see, for example, the proof of $[6$, Corollary 6]). Suppose that $S$ does not satisfy condition (a) hence not condition (b), of Theorem 1. Then there exist subsets $S_{1}, S_{2} \subseteq S$ such that $S_{1}, S_{2}$ are not left thick in $S$ but the subset $S_{1} \cup S_{2}$ is. Thus there exist finite; subsets $S_{1}^{\prime \prime}, S_{2}^{\prime \prime} \subseteq S$ such that both

$$
S_{1}^{\prime \prime} s \nsubseteq S_{1} \text {, and } S_{2}^{\prime \prime} s \nsubseteq S_{2} \text {, }
$$

for all $s \in S$. Designate $S_{1}^{\prime \prime} \cup S_{2}^{\prime \prime}$ by $S^{\prime \prime}$. Since $S^{\prime \prime}$ is finite, there exists $s^{\prime \prime} \in S$ such that $S^{\prime \prime} s^{\prime \prime}=\left\{s^{\prime \prime}\right\}$. But $S_{1} \cup S_{2}$ is left thick in $S$, so there exists an element $s^{\prime} \in S$ such that $s^{\prime \prime} s^{\prime} \in S_{1} \cup S_{2}$, which implies $s^{\prime \prime} s^{\prime} \in S_{1}$ or $s^{\prime \prime} s^{\prime} \in S_{2}$. Assume, say, the former; then

$$
S_{1}^{\prime \prime}\left(s^{\prime \prime} s^{\prime}\right) \subseteq S^{\prime \prime}\left(s^{\prime \prime} s^{\prime}\right)=\left\{s^{\prime \prime} s^{\prime}\right\} \subseteq S_{1},
$$

which contradicts (1). Hence $S$ has a multiplicative left invariant mean.

4. Examples and special cases. By use of Corollary 3, examples may be obtained of semigroups which have a multiplicative left invariant mean. These are given below.

(a) Let $S$ be a lattice where multiplication is defined by $s s^{\prime}=s \vee s^{\prime}$, for $s$, $s^{\prime} \in S$. Since $s s^{\prime \prime}=s^{\prime} s^{\prime \prime}$, where $s^{\prime \prime}=s s^{\prime}$, then $S$ has a multiplicative left invariant mean by Corollary 3.

(b) Another example is given by the semigroup of "almost fixed" maps on an infinite set $X$. That is, let $S$ be the family of those maps $s: X \rightarrow X$ which satisfy the condition that the set $Y(s)=\{x \in X ; s x \neq x\}$ is finite. Then $S$ has the required property. For let $s, s^{\prime} \in S$; denote $Y(s) \cup Y\left(s^{\prime}\right)$ by $Y$, and let $x_{0}$ be an arbitrary element $x_{0} \in X-Y$. Define $s^{\prime \prime} \in S$ by $s^{\prime \prime} x=x$ for $x \in X-Y$, and $s^{\prime \prime} x=x_{0}$ for $x \in Y$. It follows by direct computation that $s s^{\prime \prime}=s^{\prime \prime}=s^{\prime} s^{\prime \prime}$, hence we have by Corollary 3 that $S$ has a multiplicative left invariant mean.

(c) Let $X$ be an uncountably infinite set; let $S$ be the semigroup of those maps $s: X \rightarrow X$ with the property that the set $\{x \in X ; s x \neq x\}$ is finite or countably infinite. Then $S$ can be shown by a procedure similar to that of (b) to have a multiplicative left invariant mean.

Later the converse to Corollary 3 will be shown for the cases of Abelian semigroups (Theorem 3) and left cancellation semigroups (Theorem 4). It remains 
an open question as to whether the converse holds when the semigroup is not restricted to these cases.

The next theorem shows that there exists a large class of semigroups which do not have multiplicative left invariant means.

THEOREM 2. A two-sided cancellation semigroup $S$ has a multiplicative left invariant mean if and only if $S$ is the trivial group $\{i\}$.

Proof. The "if" part follows from Corollary 1 . The converse is shown first for (a) Abelian groups, then (b) groups, and finally (c) two-sided cancellation semigroups.

(a) If $S$ is an Abelian group, then $S$ is contained (isomorphically) in $S$, the Bohr compactification of the discrete group $S$ [7, Theorem 1.8.2, p. 30]. The left multiplications of the compact Hausdorff space $S$ by elements $s \in S$ map $\bar{S}$ continuously into itself. Let $S$ have a multiplicative left invariant mean; then by Theorem 1, condition (d), there exists an element $x_{0} \in S$ such that $s x_{0}=x_{0}$ for all $s \in S$. But $S$ is a group, so $s=i$ for all $s \in S$. Hence $S=\{i\}$.

(b) Let $H$ be a subgroup of a group $S$, and let $K$ be a set of representatives for right cosets of $H$ in $S$. Thus each $s \in S$ has a unique representation as a product $s=h k$, where $h \in H$ and $k \in K$. As in [1, p. 533], define an operator $T: m(H) \rightarrow m(S)$ by $(T f)(h k)=f(h)$ for $f \in m(H), h \in H$, and $k \in K$. If $\mu$ is a left invariant mean on $S$, then $T^{*} \mu$ is a left invariant mean on $H$ [4, Theorem 17.12, p. 234]. Also, it follows by a routine computation that $T(f \cdot g)=T f \cdot T g$ for $f$; $g \in m(H)$, hence $T^{*} \mu$ is a multiplicative mean on $H$ if $\mu$ is a multiplicative mean on $S$. Let $S$ have a multiplicative left invariant mean; then so has each subgroup $H$. But if $H$ is the subgroup generated by any element $s \in S$, then $H$ is Abelian, hence trivial by (a). Therefore $S=\{i\}$.

(c) Let $S$ be a two-sided cancellation semigroup which has a left invariant mean $\mu$. Then $S$ is embeddable in some group $G^{\prime}[8$, Corollary 3.6]. Let $G$ be the subgroup of $G^{\prime}$ that is generated by $S$; let $\pi$ be the restriction map $\pi: m(G) \rightarrow m(S)$ defined by $(\pi f)(s)=f(s)$ for $f \in m(G), s \in S$. Then $\pi^{*} \mu$ is a left invariant mean on $G$ [8, Proof of Theorem 3.1 and Corollary 3.2]. Now let $\mu$ be a multiplicative mean as well as left invariant. Since $\pi\left(f_{1} \cdot f_{2}\right)=\pi f_{1} \cdot \pi f_{2}$ for $f_{1}, f_{2} \in m(G)$, it follows that $\pi^{*} \mu$ is a multiplicative left invariant mean on the group $G$. Thus $G$, hence $S$, is trivial by (b).

The partial converses to Corollary 3 that were mentioned earlier can now be obtained by use of Theorem 2 .

THEOREM 3. Let $S$ be an Abelian semigroup. Then $S$ has a multiplicative left invariant mean if and only if for every $s, s^{\prime} \in S$, there exists $s^{\prime \prime} \in S$ such that $s s^{\prime \prime}=s^{\prime} s^{\prime \prime}$.

Proof. The reverse implication follows from Corollary 3. For the forward implication, we use a construction due to E. Granirer [3]. Let $S$ be any left amenable 
semigroup, let $(r)$ be a relation defined between elements of $S$ by: $s(r) s^{\prime}$ if and only if there exists $s^{\prime \prime} \in S$ such that $s s^{\prime \prime}=s^{\prime} s^{\prime \prime}$. Then $(r)$ is a two-sided stable equivalence relation (i.e., a congruence) [3, Lemma 2, p. 371]. Let $\eta(s)=\bar{s}$ for $s \in S$, where $\bar{s}$ is the equivalence class of $S$ (with respect to $(r)$ ) that contains $s$; then $\eta$ is a homomorphism of $S$ onto the factor semigroup $S /(r)$ by $[5, \S 2.5$, p. 266]. And by $[5, \S 6.3$, p. 294$], S /(r)$ satisfies the right cancellation law:

$$
\left(\bar{s}_{1} \bar{s}_{3}=\bar{s}_{2} \bar{s}_{3}\right) \rightarrow\left(\bar{s}_{1}=\bar{s}_{2}\right)
$$

for $\bar{s}_{1}, \bar{s}_{2}, \bar{s}_{3}, \in S /(r)$.

Now let $S$ be an Abelian semigroup and let $S$ have a multiplicative left invariant mean. By the paragraph above, $\eta(S)=S /(r)$ where $\eta$ is a homomorphism, so $S /(r)$ has a multiplicative left invariant mean by Corollary 2 . But $S /(r)$ is a right cancellation semigroup, hence a two-sided cancellation semigroup since $S /(r)$ is Abelian. Therefore $S /(r)$ consists of one element by Theorem 2, which means that any two elements $s, s^{\prime} \in S$ satisfy; the relation $s(r) s^{\prime}$, thus showing Theorem 3.

We remark that the technique that was used in the proof of the above theorem runs into difficulty if the condition that $S$ is Abelian is dropped. For in such a case, $S /(r)$ is a right cancellation semigroup. We must show that $S /(r)$ is also a left cancellation semigroup. But we have been unable to do this without imposing additional conditions on $S$. However, the proof goes through without difficulty if the requirement that $S$ is a left cancellation semigroup is added. For if $S$ satisfies a left cancellation law, then it follows by direct computation that so does $S /(r)$, and thus we have by a proof similar to that of the previous theorem:

THEOREM 4. Let $S$ be a semigroup with left cancellation. Then $S$ has a multiplicative left invariant mean if and only if for every $s, s^{\prime} \in S$, there exists $s^{\prime \prime} \in S$ such that $s s^{\prime \prime}=s^{\prime} s^{\prime \prime}$.

Addendum. Since the completion of this paper, E. Granirer (Extremely amenable semigroups, to appear) has resolved the open question mentioned in $\$ 4$ by showing that Theorem 3 still holds if the assumption that $S$ is Abelian is dropped. This implies that each semigroup with the common fixed point property on compacta is of the class $\pi$, which I had conjectured to be false. I am indebted to E. Granier for providing me with a pre-print of his paper.

\section{REFERENCES}

1. M. M. Day, Amenable semigroups, Illinois J. Math. 1 (1957), 509-544.

2. - Fixed-point theorems for compact convex sets, Illinois J. Math. 5 (1961), 585-590.

3. E. Granirer, A theorem on amenable semigroups, Trans. Amer. Math. Soc. 111 (1964), 367-379.

4. E. Hewitt and K. A. Ross, Abstract harmonic analysis. I, Grundlehren der Mathematischen Wissenschaften, Bd. 115, Academic Press, New York, 1963. 
5. E. S. Ljapin, Semigroups, Transl. Math. Monos. Vol. 3, Amer. Math. Soc., Providence, R. I., 1963.

6. T. Mitchell, Constant functions and left invariant means on semigroups, Trans. Amer. Math. Soc. 119 (1965), 244-261.

7. W. Rudin, Fourier analysis on groups, Interscience Tracts in Pure and Applied Mathematics, Vol. 12, Interscience, New York, 1962.

8. C. Wilde and K. Witz, Invariant means and the Stone-Čch compactification, Pacific Math. Soc. (to appear).

STATE UNIVERSity OF New YoRK, Buffalo, New YoRK 